\title{
Measurement of Renal Dimensions to Determine Standard Reference Renal Volume Model for Clinical Application in Ghana Issahaku Shirazu $^{1}$, Y. B Mensah ${ }^{2}$, Cyril Schandorf ${ }^{3}$, S. Y. Mensah ${ }^{4}$
}

\author{
${ }^{1}$ University of Cape Coast, School of Agriculture and Physical Sciences Faculty of Physical Sciences, Department of Physics, \\ Cape coast, Ghana \\ ${ }^{3}$ Graduate School of Nuclear and Allied Sciences, University of Ghana, Legon, Ghana \\ ${ }^{2}$ University of Ghana Medical School, Department of Radiology, Korle-Bu Teaching Hospital, Accra Ghana \\ ${ }^{4}$ University of Cape Coast, School of Agriculture and Physical Sciences, Faculty of Physical Sciences, Department of Physics \\ Cape Coast, Ghana
}

\begin{abstract}
The study is based on measurements of renal dimensions to determine standard reference renal volume model and renal volumetric ellipsoid coefficient. Two methods, Voxel count method and rotational renal ellipsoid equation were the two measuring tools used with an integrated MVL application software platform. The procedure involve measurement of linear dimensions together with using the snake technique to draw the region of interest (ROI) for the volumetric measurements. These were done to obtain: renal length, lateral diameter, A-P diameter and the total number of voxels to estimates the renal volume. These parameters were used to calculate renal volume using the rotational renal ellipsoid equation as well as using the Minitab statistical software to model renal volume equation. The reference standard renal volume was also determined using water displacement with the Archimedes' principle. The average renal shape index and its dispersion (i.e. covariance matrix) was approximately $1 \pm 0.02$. The male average measured values for right and left kidneys were as follows: renal length, $103.35 \mathrm{~cm}$ and $105.13 \mathrm{~cm}$, lateral diameter, 60.79 and 60.40 and A-P diameter, 44.12 and 44.95 and renal volume; $146.74 \mathrm{~cm} 3$ and $151.76 \mathrm{~cm} 3$ respectively. Furthermore, the female average measured values for right and left kidneys were as follows: renal length, 101.43 and 102.98, lateral diameter, 59.20 and 59.02 and A-P diameter, 43.09 and 44.82, renal volume $142.04 \mathrm{~cm} 3$ and $148.29 \mathrm{~cm} 3$ respectively. The average estimated renal volumetric ellipsoid coefficient $(\mathrm{VeC})$ was 0.53 . However, various variations for both male and female, with their corresponding right and left kidneys were, $0.5283,0.5297,0.5280$ and 0.5304 respectively. The reference renal dimensions including the standard renal volume and renal volumetric ellipsoid coefficient are recommended for clinical application in Ghana.
\end{abstract}

Keywords : Renal volumetric ellipsoid coefficient, renal shape index, renal volume, rotational ellipsoid equation, MVL DICOM

\section{INTRODUCTION}

Medical imaging is described as the method for noninvasive assessment of physiological and anatomical information about human tissues or organ, by analysing patterns observed within the imaged tissues or organ [1]. In addition, the analysis and interpretation of these pattern for diagnostic and or therapeutic decisions are based on observable pattern of various parameters which a trained clinician looks for: these manifest as morphological and functional changes. Some useful parameters of the acquired image in radiological examinations include volumetric and linear measurements. These parameters enable differentiation between normal and abnormal tissues by the differential radiation dose distribution based on their morphological and contrast variations [1]. These patterns are used to determine whether tissues are benign or malign, and intact or repaired. Medical imaging can thus be used to test the effectiveness of remedies and evaluate the effects of treatments for specific diseases and are display in the form of organ model [2].

These organ models have gradually gained prominence in medical image analysis and clinical research. $[1,2]$. Furthermore, these models are used to establish standard reference values that aid radiologists, nuclear medicine 
experts and oncologists to make important diagnostic and therapeutic decisions by comparing patient's organ parameters to these reference values. [2]. In addition, modeled standard reference values like renal volume provides more accurate assessment of the renal size than the traditional method of using renal length to estimate renal size without available baseline reference values [2]. The establishment of basic radiological reference linear renal dimensions, like, renal shape index, Anterior-Posterior (A-P), longitudinal and lateral diameters are important for the diagnosis and prognostic evaluation of nephropathies. In addition, these linear dimensions are also use to design area and volume models. Indeed, the reference information from the designed models can be used as standard to test future research data as well as to explain anatomic variations between individuals of the same population and across populations [3].

In applied physics, well-developed human organ system models are described by mathematical expressions [1] These expressions are converted to computer aided designed (CAD) models for physical visualized analysis, in terms of visual indicators and graphic user interface (GUI) for clinical application [1]. Currently, image assisted construction of organ models are design using 6D ( $\mathrm{x}, \mathrm{y}, \mathrm{z}$, time, colour and user define) volume element (voxel) model of various reconstructed images, based on their varied grey level values. The differentiation of the grey value areas in these organs are done by the process of image segmentation $[4,5]$. In the case of CT scan, gray values represented by the Hounsfield numbers are replaced by the organ identification numbers in a container called voxel. This voxels are describe by a single data point, representing a regularly spaced in three-dimensional grid. This may present a single piece of data, such as an opacity or a multiple pieces of data such as opacity and color. Depending on the type of data and the intended use of the dataset, several voxels can be reconstructed or approximated through interpolation to form an entire volume [6]. In view of this voxel are used in medical image analysis to represent the smallest 3D unit of various organs volume, which are estimated from the pixel size in addition to the slice thickness.

There are two ways by which voxel representation of the structures of human organs models can be done; these are; the statistical shape model and the statistical appearance model [7]. The statistical shape model represents the shape information, such as renal dimensions (A-P diameter, renal length and lateral diameter), renal volume and renal surface area $[1,7]$. These are regarded as the most useful tools for studying variations in anatomical shape of organs and has been widely used in medical image analysis, such as, medical image segmentation, shape registration and interpretation [1].

\section{A. Objectives}

The aims of the study is to

Measure renal dimensions in order to determine standard reference renal dimensions and volume models for clinical application.

Determine renal volumetric ellipsoid coefficient and renal shape index to predict individual renal volume using ellipsoid equation for clinical application.

Reviewed and compare the established renal dimension and renal volume estimates with international recommendations and reference values and make appropriate recommendations.

\section{B. Literature Review}

A number of publications appeared in literature in an attempt to describe renal dimensions and other related parameters by various institutions and organizations. Most of these studies are based on measurements of longitudinal diameter, anterior-posterior (A-P) diameter, transverse diameter, renal surface area and renal volume. These parameters provided standardized range of estimates of normal renal sizes by these organizations and individuals [8-28]. However, African clinicians and researchers are yet to be part of these significant developments.

Various studies estimated renal volume using a known pixel size and a slice thickness for computation. A number of studies to measure renal dimensions in order to predict kidney size concluded that longitudinal diameter varied between $10.2 \mathrm{~cm}$ to $11.8 \mathrm{~cm} \pm 2.3 \mathrm{~cm}$ on the left and $9.8 \mathrm{~cm}$ to $10.9 \mathrm{~cm} \pm 2.1 \mathrm{~cm}$ on the right. Transverse diameter varies between $58.0 \mathrm{~cm}$ to $6.25 \pm$ $0.67 \mathrm{~cm}$ on the right and from 6.01 to $6.43 \pm 1.7 \mathrm{~cm}$ on the 
right and A-P diameter varies between $4.06 \mathrm{~cm}$ to $4.73 \mathrm{~cm} \pm 0.65 \mathrm{~cm}$ on the right 4.15 to $4.88 \pm 0.95 \mathrm{~cm}$ on the left kidney. It has also been reported that renal volumes varies between $132-196 \pm 35.05 \mathrm{~cm}^{3}$ for the left kidney and $128.4 \mathrm{~cm}^{3}$ to $194 \pm 28.17 \mathrm{~cm}^{3}$ for the right kidney for male. The studies also shows that the female kidney volume varies between $134 \mathrm{~cm}^{3}$ to $186.5 \pm 23.56$ $\mathrm{cm}^{3}$ for right kidneys with corresponding left kidney volume estimated to varies between $136 \mathrm{~cm}^{3}$ to $193.1 \pm 34.77 \mathrm{~cm}^{3}$ [15]. Another radiological organ measurements shows that normal adult renal weight varies between 173.0 to $196.3 \pm \mathbf{4 1 . 0} \mathrm{g}$ [15]. Most of the study concluded by predicting that kidney sizes diminishes with advancing age, due to parenchymal reduction of the kidney [16].

\section{METHODS AND MATERIAL}

\section{A. Materials}

The material used include: The Vernier caliper (Figure 1) was used to measure the dimensions of the kidney and the Triple-beam balance (Figure 2) to measure the weight. The beaker for the application of the Archimedes principle of volume measurement. Five different types of MDCT Machine (Figure 3), with slices variation of between 16 slice to 640 slice. The images (Figure 4 ) that met the selection criteria were copied onto DVD and transfer onto the the MVL aplication workstation (Figure 4). The MVL user interface enable it to be implemented in any advance computer system.

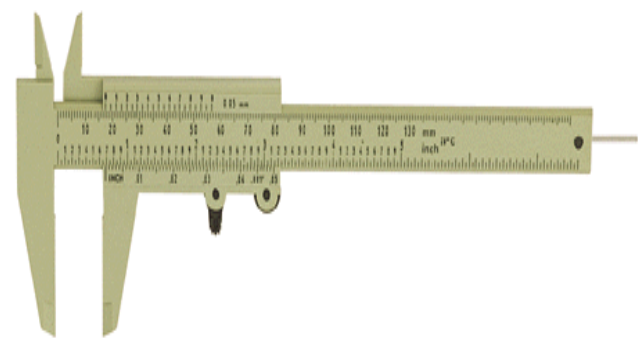

Figure 1. Vernier Caliper

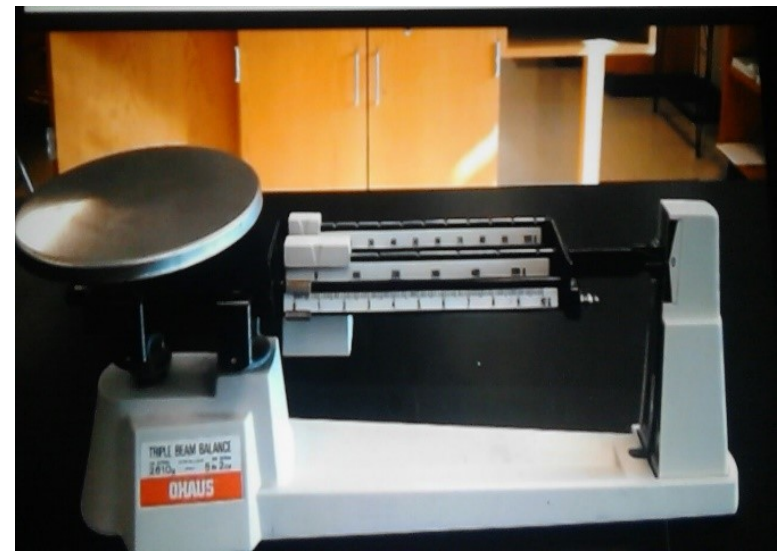

Figure 2. Triple-Beam Balance

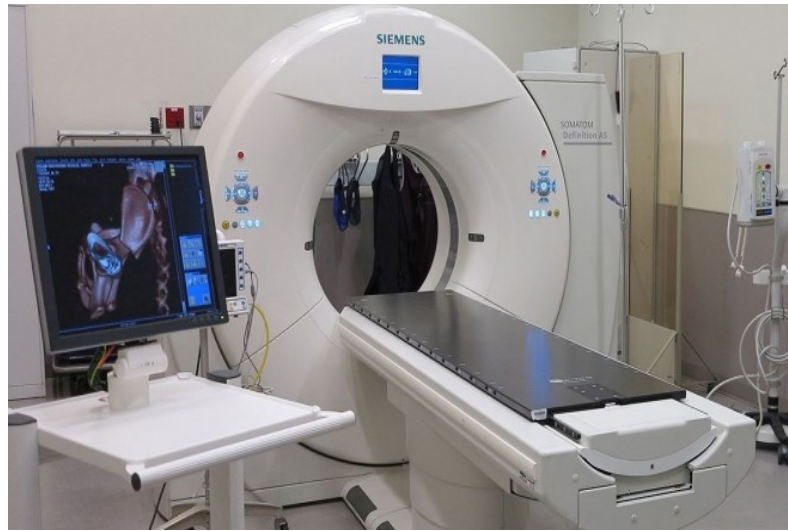

Figure 3. MDCT Machine

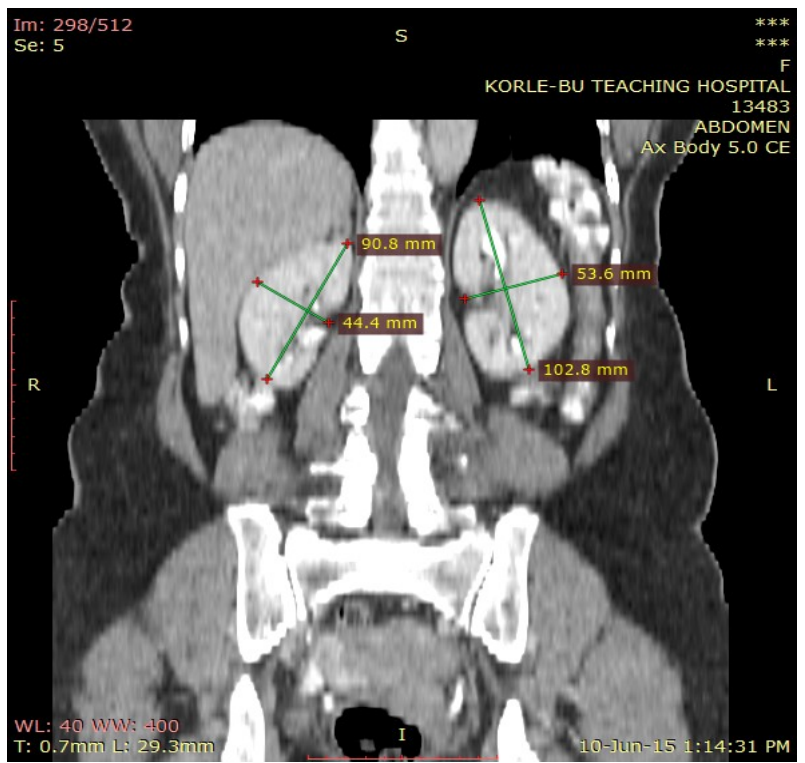

Figure 4. Image For Linear Dimensions

\section{B. Methodology}

The activities before measurements include: 
$>$ Identified and code images that met the selection criteria for transfer on to the MVL application platform.

$>$ Reconstruct images at $5 \mathrm{~mm}$ to unify the measuring process in all the $\mathrm{CT}$ centers.

$>$ The images were then copied from the image archiving and storage system called the PACS from all the 10 CT centers onto DVD and transferred onto MVL application platform and ready for measurements.

\section{Measurements of Linear Renal Dimensions}

The first to be measured were the three linear dimensions, described as longitudinal diameter or the renal length, transverse diameter or the lateral diameter (renal width) and the A-P diameter (renal thickness). All kidney dimensions were measured at maximum values of strictly longitudinal, Anterior-Posterior and transverse sections through the center of the kidney. The renal length were measured using the coronary images while the axial images were used to measure A-P and lateral diameters. The width and thickness were measured in the transverse plane perpendicular to each other and the longitudinal axis of the kidney. The level of this transverse section was placed at the level of the hilum.

Two different methods were used to measure longitudinal diameter (LNG) on the coronary images: The first method of measurements was done by drawing a single straight line from one edge of the renal parenchyma to another end with the application software tool on the MeVisLab (MVL) platform as shown in Figure 5. This was repeated three times and average of the three measured values calculated as the renal length. Secondly, the renal length was calculated from axial slices by multiplying the slice thickness by the number of slices between the superior and inferior tips of the kidneys. This were also repeated three times and the average value estimated.

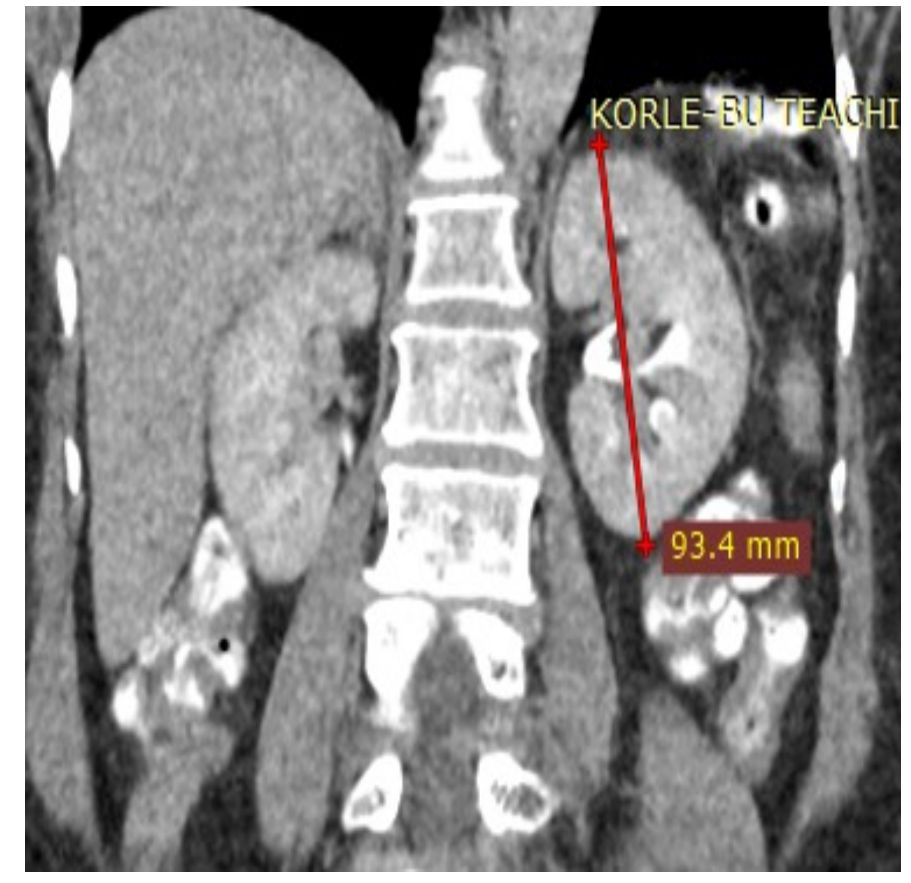

Figure 5: Renal Length

In addition, two other linear parameters were measured: the lateral diameter (LT), measured from the lateral extent of the kidney to the renal sinus and anteriorposterior (A-P) diameter measured perpendicular to the lateral diameter as shown in Figure 3.6. The two measurements were repeated three times and the average values of both parameters estimated to represent the average lateral and A-P diameters.

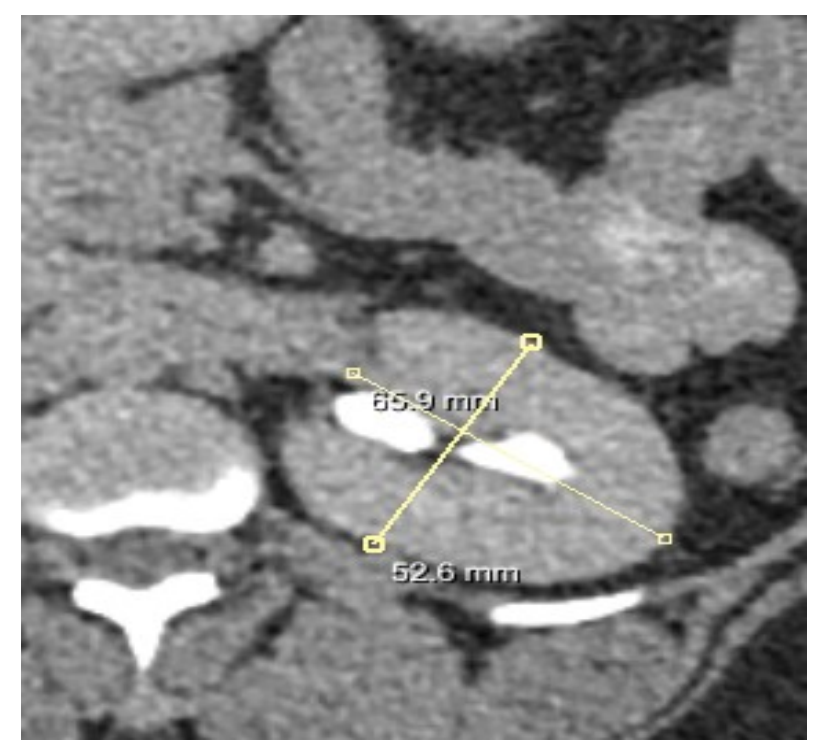

Figure 6. Lateral and A-P diameters 


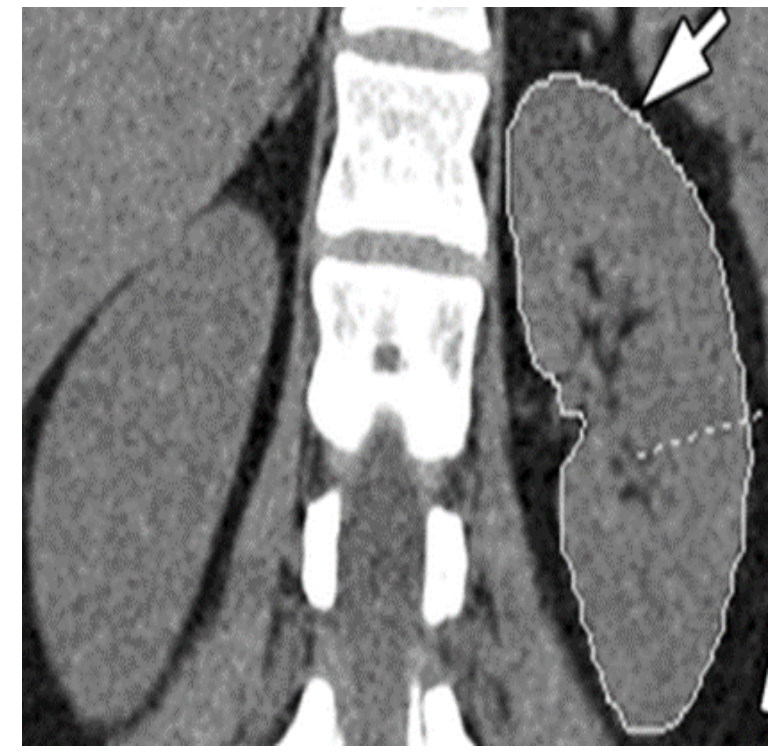

Figure 7 Renal Surface Area

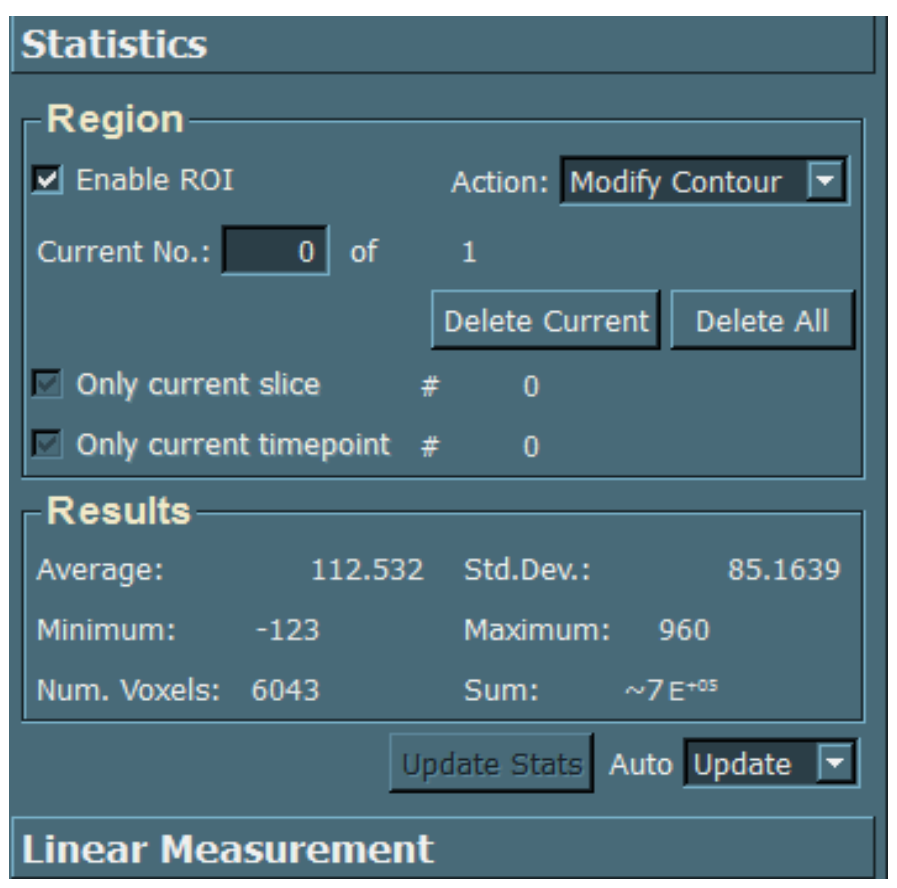

Figure 8. Measurements of RSA and RSI

\section{Measurements of Renal Volume}

The second component of the study was the measurements of renal volume from contiguous CT slices with voxel measuring tool on the MVL application software as shown in Figure 8. These measurements were done using $3 \mathrm{D}$ volume-rendered image of the kidney shown in Figure 3.4. The maximum length of the kidney was measured in the longitudinal plane and was visually estimated to represent the largest longitudinal section. Two different methods were used to calculate renal volumes.
The first method was the calculation of the total renal volume by using the voxel-count method on the MVL application software. With a region of interest (ROI) drawn on each of the two kidneys on each slice to indicate the renal boundaries. The total voxel was automatically generated on each slice by taking the sum of the voxel lying within the boundaries, including the central sinus fat but excluding perinephric fat as much as possible.

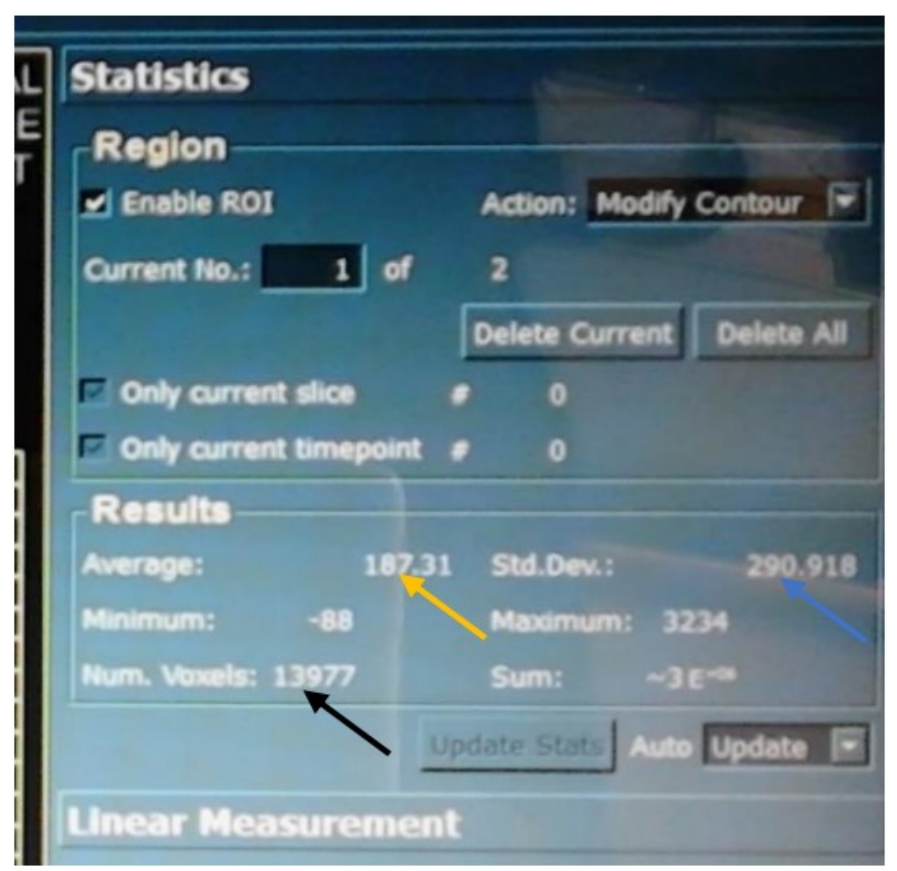

Figure 9. Automated calculated of total number of voxel count.

With a known pixel size, slice thickness and the total number of voxels (as shown in Figure 9 with black arrow). The renal volumes were computed for each patient using the equation below.

That is, $R V=$ Total number of voxels $\mathrm{x}$ slice thickness $\mathrm{x}$ pixel length $\mathrm{x}$ pixel width.

The advantage of using this method is that the shape of the kidney is irrelevant during measurements. An average of three voxel-count measurements and three other repeated measurements was used as the referencestandard renal volume.

Indeed, this method of volume measurement may result in partial voluming, which occurs when voxels contain both kidney and surrounding tissue, could lead to an overestimation of the renal volume when all such voxels 
are included within the boundaries of the kidney. To avoid this overestimation, the segmentation line was drawn at the halfway point of the change in signal intensity, between the kidney and the surrounding tissue and use as reference measuring point in all the slices.

A second method to estimate renal volume was by the Archimedes principle. Here renal volume was estimated using the fluid displacement principle refers to, as Archimedes principle, which states that; the volume of displaced fluid is equivalent to the volume of an object fully immersed in a fluid or to that fraction of the volume below the surface for an object partially submerged in a fluid. The volume and mass of the kidneys were measured from Cadavers during autopsy on fresh dead bodies. The process involve clamping the kidneys before anastomosis and slowly lowered the kidney into a large glass beaker placed on a Triple-beam balance and the displayed volume estimated. Here a large glass beaker was placed on the Triple-beam balance (Figure 10) and calibrated by aligning the reading to the zero mark and the spring elongation ensure to read zero as well. The beaker was then fill with water to about $3 / 4$ capacity and placed on the pan of the triple-beam balance. The triple-beam balance was then recalibrated or realigned to the initial line by moving the moveable masses.

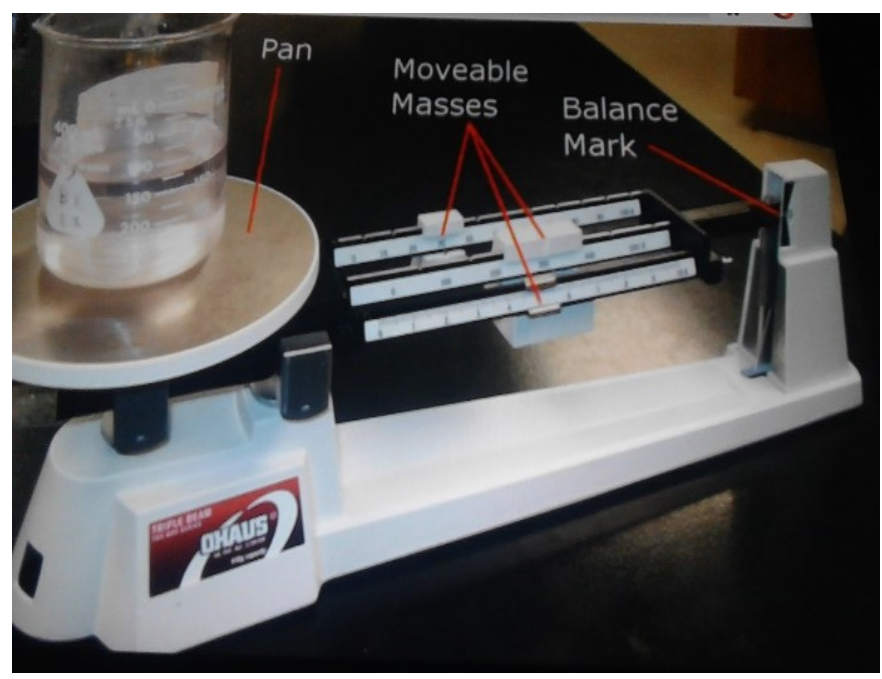

Figure 10. Large Metal Beaker Fill With Water On Triple-Beam Balance.

Next, the kidney was lowered and completely submerged in the water and the volume determined, by finding the difference between the initial volume of the water reading and the displaced volume reading. An average of three readings were taken and mean value estimated. At the same time, the difference between the total combine weight of the beaker and the water from the total combined weight of the beaker, water and the kidney represent the renal weight. The average of three repeated measured values was determined as the renal weight.

\section{E. Measurements of Renal Parameters}

Four important parameters associated with the linear renal dimensions and volume measurements were also estimated. These include: Renal shape index (RSI), renal surface area (RSA), relative renal length (RRL) and relative renal volume (RRV). First, the renal shape index was estimated by using the ratio of the renal length to the sum of lateral and A-P diameters.

Mathematically, RSI is estimated as:

$$
\text { RSI }=\frac{\text { renal length }}{\text { renal width }+ \text { renal thickness }}
$$

That is with known A-P diameter, renal length and the lateral diameter, equation 1 was used to estimate the renal shape index. Secondly, renal surface area was estimated by using the MVL application software tool to map out the kidney contour on a slice that contain the total surface area of the kidney as shown with white arrow on Figure 7. This was done by manually tracing the boundaries of the surface area of the kidney on the slice that contain the complete renal surface. The total number of pixels were automatically generated (Figure 3.9) by MVL software based on the region of interest (ROI) lying within the surface boundaries of the kidney. The measurements were repeated three time and the average value estimated. With the known pixel size and the total voxels, RSA was estimated. This was done using the relation

\section{RSA $=$ pixel size $x$ number of total pixels}

Furthermore, two relative renal parameters were also estimated and describe as relative renal length and relative renal volume. The relative renal length (RRL) was determined by dividing either the right renal length $\left(R R_{L}\right)$ or the left renal length $\left(L_{L}\right)$ by the total renal length (sum of the left renal length and right renal length). In addition, the relative renal volume (RRV) 
was also estimated by dividing either the right renal volume $\left(R_{V}\right)$ or the left renal volume $\left(L_{V}\right)$ by the total renal volume (sum of right renal volume and left renal volume).

$$
\begin{gathered}
R R L=\frac{R R_{L}}{R R_{L}+L R_{L}} \\
R R V=\frac{R R_{V}}{R R_{V}+L R_{V}}
\end{gathered}
$$

The renal volumetric ellipsoid coefficient, was estimated by dividing the measured renal volume by the product of renal length, renal width and renal thickness. It represent the constant of proportionality in the ellipsoid equation The K-values were determine by using the ellipsoid equation for estimating renal volume define as:

$\mathbf{R V}=\mathbf{K} *$ renal length $(\mathbf{R L}) *$ renal thickness $(\mathbf{R T}) *$ renal width $(\mathbf{R W})$. Implied,

$$
K=\frac{R V}{R L * R T * R W}
$$

Therefore with a known renal volume by the voxel count method, renal length, renal width and renal thickness by linear measurements by MVL. Then $\mathbf{K}^{*}$ was estimated and the standard reference ellipsoid equation with known $\mathbf{K}^{*}$ define as:

$$
\boldsymbol{R V}=\boldsymbol{K}^{*} * \boldsymbol{R} \boldsymbol{L} * \boldsymbol{R T} * \boldsymbol{R} \boldsymbol{W}
$$

\section{RESULTS AND DISCUSSION}

The basic relationship between the various parameters in tables and graphical representation are discussed here. Presentation of the summarized data and the analysis are shown below. All the measured primary data parameters are in the unit of $\mathrm{mm}$, unless otherwise stated.

\section{A. RESULTS}

GRAPHICAL REPRESENTATION

\section{AGE AND GENDER VARIATION OF $\mathrm{RL}$}

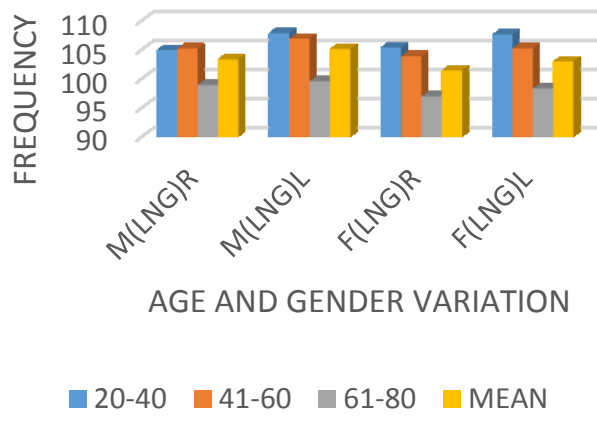

Figure 11. Age and gender variation of Renal Length AGE AND GENDER VARIATION OF RV

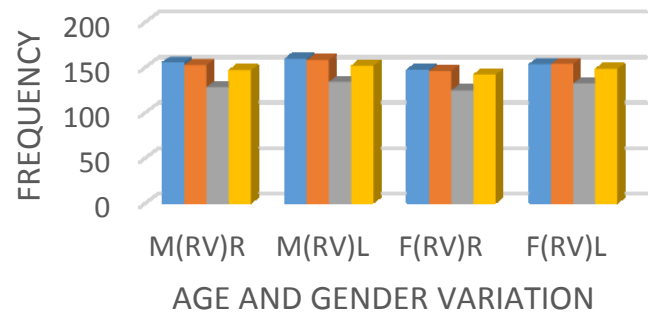

-20-40 41-60 $\square$ 61-80 $\square$ MEAN

Figure 12. Age and gender variation of Renal Volume

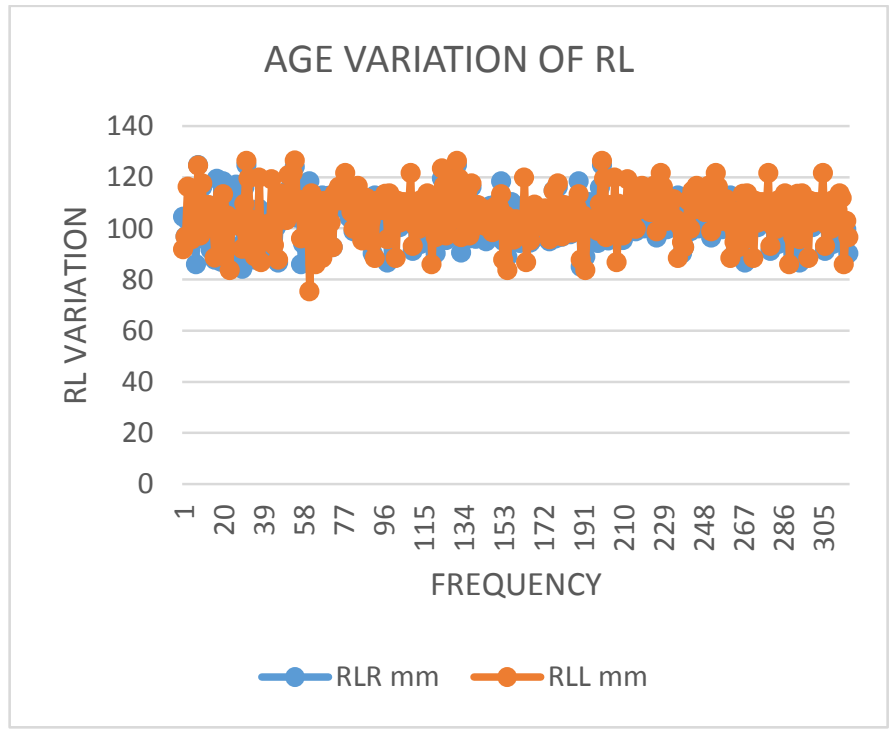


Figure 13. Male age variation of Renal Length

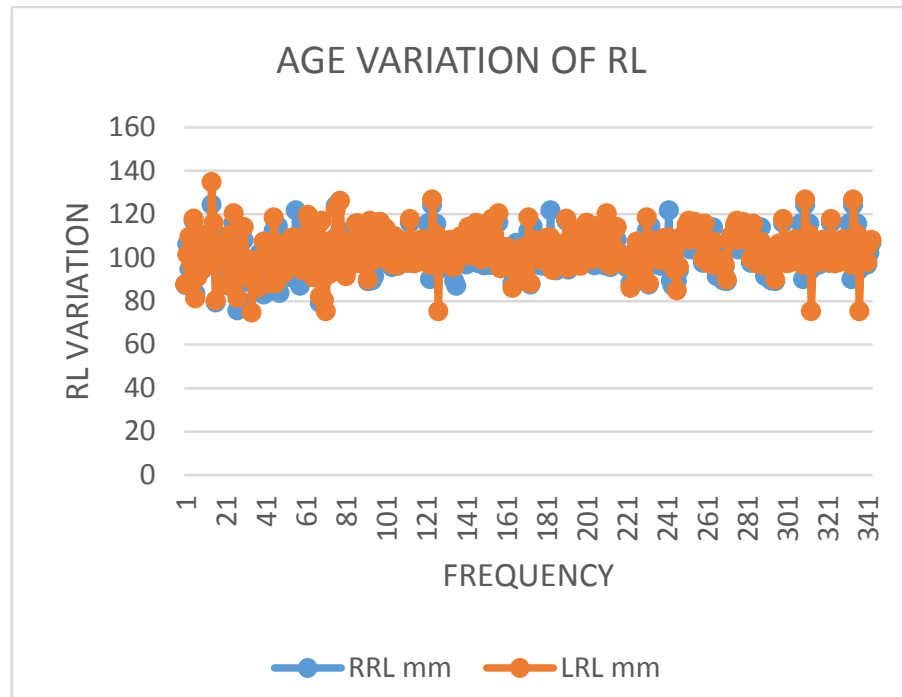

Figure 14. Female age variation of Renal Length

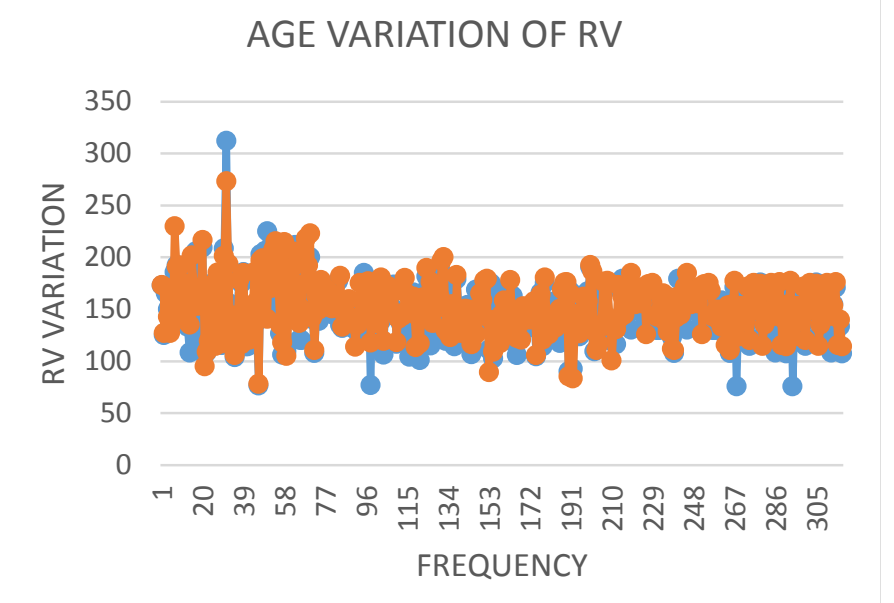

$$
\longrightarrow \text { RVR } \mathrm{cm} 3 \longrightarrow \text { RVL cm3 }
$$

Figure 15. Male age variation of Renal Volume

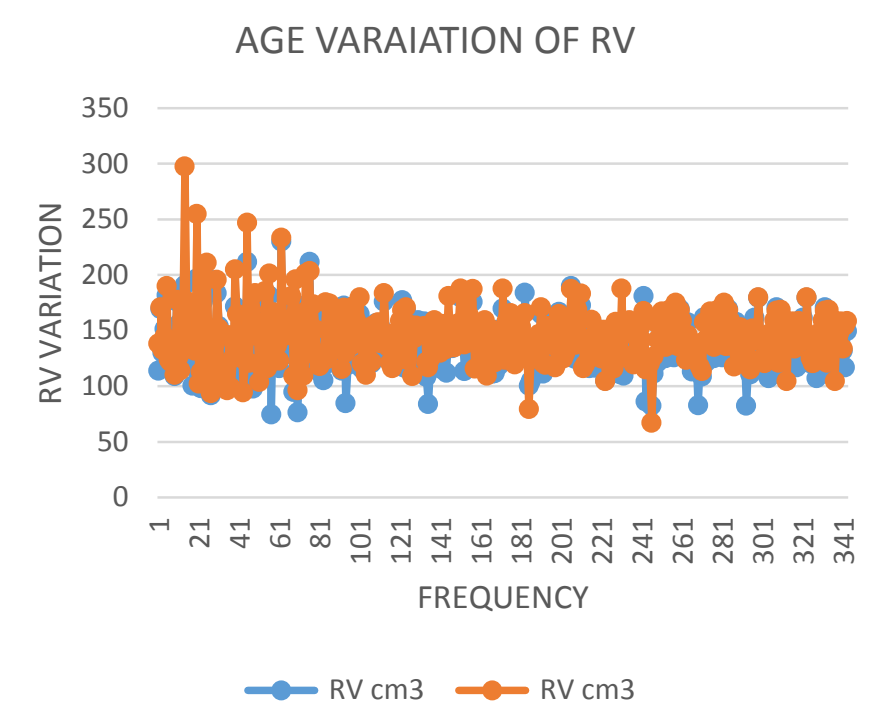

Figure 16. Female age variation of Renal Volume

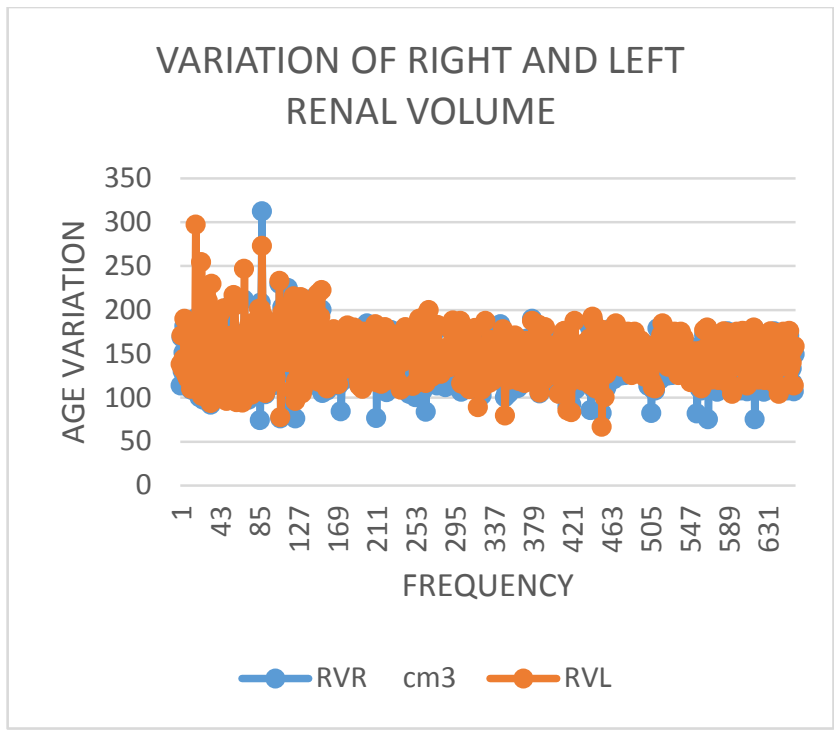

Figure 17. Age variation of Renal Volume VARIATION OF RIGHT AND LEFT RENAL
LENGTH

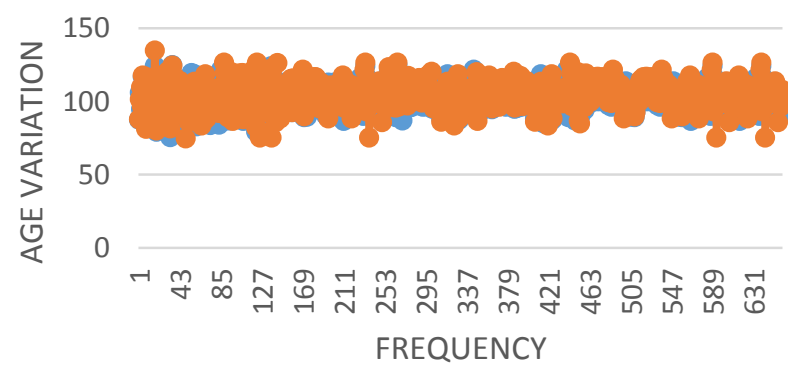

$\longrightarrow$ LNGR $\longrightarrow$ LNGL

Figure 18. Age variation of Renal Length RELATIONSHIP BETWEEN RENAL VOLUME AND RENAL LENGTH

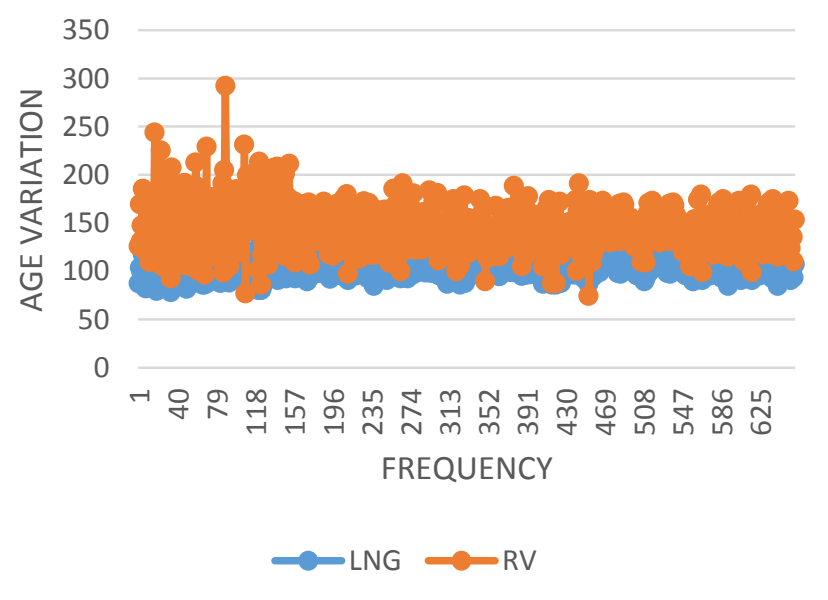

Figure 19. Age variation of Renal Length and Renal Volume 


\section{B. Regression Analysis}

Determination of RV Using $\mathrm{R}_{\mathrm{e}}$ (Male Linear Renal Dimension)

Model Equation

$$
\mathrm{RV}=\mathbf{0 . 5 3} \mathrm{R}_{\mathrm{E}}+\mathbf{1 . 1 9}
$$
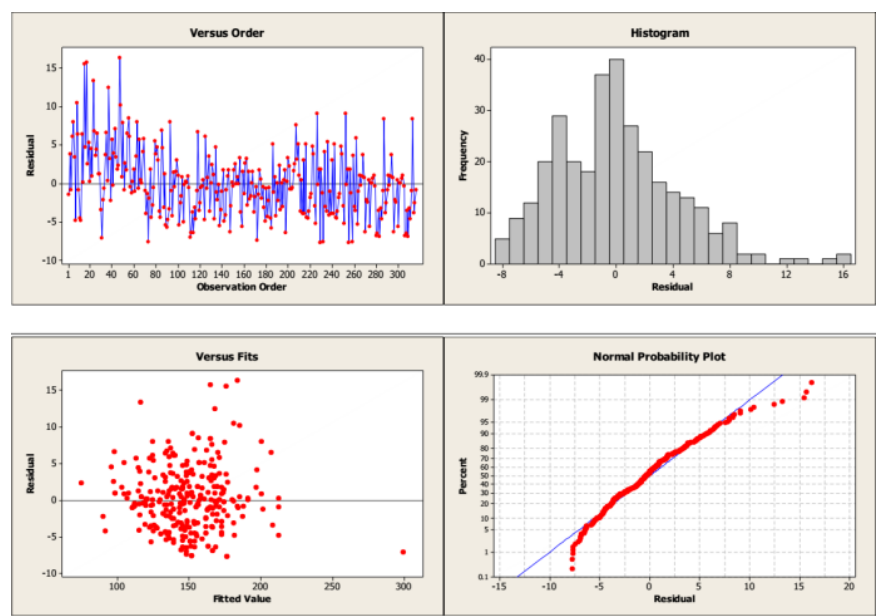

Figure 20. Renal volume in relation to Re variations for age and gender

\section{Determination of RV Using $R_{e}$ (Female Linear Renal} Dimension)

\section{Model Equation}

$$
\mathrm{RV}=\mathbf{0 . 5 2} \mathrm{R}_{\mathrm{E}}+\mathbf{1 . 8 1}
$$

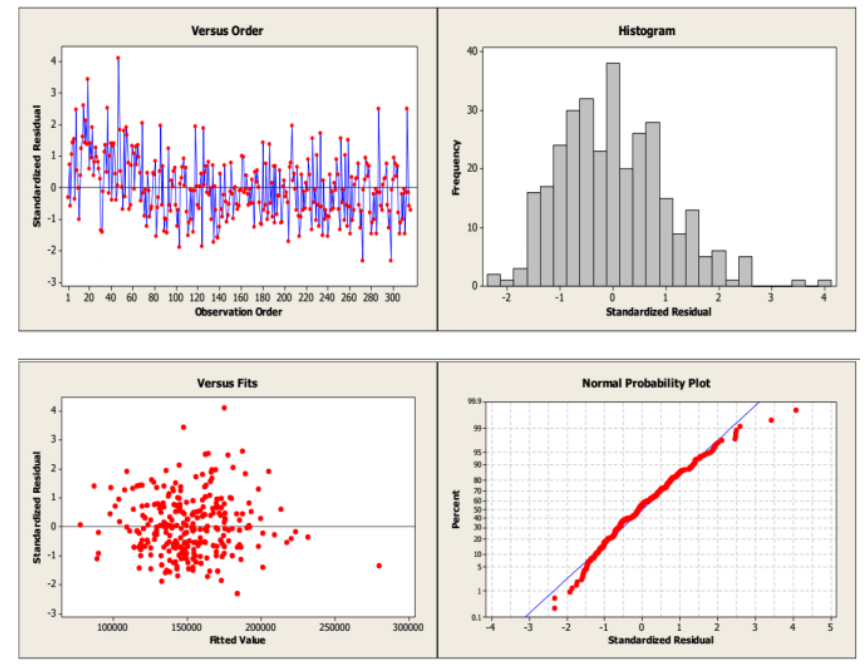

Figure 21. Renal volume in relation to Re variations for age and gender
D. Model Ellipsoid Equation

MALE ELLIPSOID EQUATION

$20-40 \boldsymbol{R} \boldsymbol{V}=\mathbf{0 . 5 2 8 7} * \boldsymbol{R} \boldsymbol{L} * \boldsymbol{R} \boldsymbol{T} * \boldsymbol{R} \boldsymbol{W}$

$41-60 \boldsymbol{R} \boldsymbol{V}=\mathbf{0 . 5 3 0 0} * \boldsymbol{R} \boldsymbol{L} * \boldsymbol{R T} * \boldsymbol{R W}$

$61-80 \boldsymbol{R} \boldsymbol{V}=\mathbf{0 . 5 2 9 5} * \boldsymbol{R} \boldsymbol{L} * \boldsymbol{R T} * \boldsymbol{R W}$

(7) FEMALE ELLIPSOID EQUATION

$20-40 \boldsymbol{R V}=\mathbf{0 . 5 2 8 8} * \boldsymbol{R} \boldsymbol{L} * \boldsymbol{R} \boldsymbol{T} * \boldsymbol{R} \boldsymbol{W}$

$41-60 \boldsymbol{R} \boldsymbol{V}=\mathbf{0 . 5 2 9 0} * \boldsymbol{R} \boldsymbol{L} * \boldsymbol{R} \boldsymbol{T} * \boldsymbol{R W}$

$61-80 \boldsymbol{R V}=\mathbf{0 . 5 2 9 5} * \boldsymbol{R L} * \boldsymbol{R} \boldsymbol{T} * \boldsymbol{R} \boldsymbol{W}$

MEAN FEMALE RENAL ELLIPSOID EQUATION:

$\boldsymbol{R} \boldsymbol{V}=\mathbf{0 . 5 2 9 2} * \boldsymbol{R L} * \boldsymbol{R T} * \boldsymbol{R W}$

MALE RENAL ELLIPSOID EQUATION:

$\boldsymbol{R V}=\mathbf{0 . 5 2 9 0} * \boldsymbol{R L} * \boldsymbol{R T} * \boldsymbol{R W}$

E4.1M

FEMALE RENAL ELLIPSOID EQUATION:

$\boldsymbol{R} \boldsymbol{V}=\mathbf{0 . 5 2 9 2} * \boldsymbol{R L} * \boldsymbol{R T} * \boldsymbol{R} \boldsymbol{W}$

E4.1F

GENERAL RENAL ELLIPSOID EQUATION

$\boldsymbol{R V}=\mathbf{0 . 5 3} * \boldsymbol{R L} * \boldsymbol{R T} * \boldsymbol{R W}$

E4.3A

D. Discussions

Demographic Statistics

This section deals with the exploratory and inferential statistical analysis of the data obtained from the total sample population. The analysis focuses on the detailed description of the obtained data with respect to certain demographic factors. These include age and gender variation of renal and body parameters in relation to exposure and dose parameters based on the various standard acquisition protocols. The measured parameters were based on the population distribution of the sample population of Ghana, as presented by Ghana statistical service department [20]. Summary of the sample demography are shown in Table 4.1. 
Table 1. Sex And Age Distribution of Data.

\begin{tabular}{|l|l|r|r|r|}
\hline Age group & Gender & Sample size & No of Kidneys & Percentage (\%) \\
\hline $20-40$ & Male & 108 & 216 & 61.71 \\
\hline & Female & 67 & 134 & 38.29 \\
\hline & TOTAL & 175 & 350 & 100 \\
\hline $41-60$ & Male & 119 & 238 & 46.12 \\
\hline & Female & 139 & 276 & 53.88 \\
\hline & TOTAL & 258 & 516 & 100 \\
\hline $61-80$ & Male & 89 & 178 & 39.21 \\
\hline & Female & 138 & 276 & 60.79 \\
\hline & TOTAL & 227 & 454 & 100 \\
\hline $20-80$ & Male & 316 & 632 & 47.88 \\
\hline & Female & 344 & 688 & 52.12 \\
\hline & TOTAL & 660 & 1320 & 100 \\
\hline & & & & \\
\hline
\end{tabular}

\section{Renal Parameters}

Base on the selection criterion only images with two kidneys were measured and analyzed and presented as left and right kidneys to the spine. In all, six renal parameters ( 3 measured and 3estimated) were analyzed. The male renal dimensions on the right of the spine are tabulated in Table 2.

Three other important renal parameters were estimated, these include: renal volume (RV), renal shape index (RSI) and renal volumetric ellipsoid coefficient (VeC). Highlight of the summarized statistics are shown in Table 3.

Renal volume is an important determinant factor during renal development, the hypothesis of various renal dimensions were tested with ages (20 to 80 years) and gender (male and female) variations. The period between 20 to 80 years is considered significant as it followed renal developmental pattern.
Table 2. Summary of Renal Dimensions

\begin{tabular}{|l|l|l|l|l|l|l|r|r|}
\hline SEX & Age & Sex & A-Pr & A-PL & TRNR & TRNL & LNGR & LNGL \\
\hline & Yrs. & M/F & mm & mm & mm & mm & mm & Mm \\
\hline MALE & Mean & Sample & & & & & & \\
\hline $21-40$ & 53 & 108 & 45.39 & 45.32 & 62.25 & 61.71 & 104.94 & 107.86 \\
\hline $41-60$ & 33 & 119 & 44.59 & 45.60 & 61.80 & 61.14 & 105.21 & 106.90 \\
\hline $61-80$ & 70 & 89 & 42.03 & 43.64 & 57.74 & 57.85 & 98.95 & 99.57 \\
\hline MEAN & 49 & 316 & 44.12 & 44.95 & 60.79 & 60.39 & 103.35 & 105.13 \\
\hline $20-60$ & & & $1.80 \%$ & -0.6 & $0.72 \%$ & $0.90 \%$ & $-0.30 \%$ & $0.90 \%$ \\
\hline $40-80$ & & & $5.70 \%$ & $4.40 \%$ & $6.60 \%$ & $5.40 \%$ & $6.00 \%$ & $6.90 \%$ \\
\hline $\begin{array}{l}\text { FEMAL } \\
\text { E }\end{array}$ & Mean & Sample & & & & & & \\
\hline $21-40$ & 34 & 67 & 43.46 & 45.22 & 60.17 & 59.19 & 105.41 & 107.63 \\
\hline $41-60$ & 53 & 138 & 44.02 & 45.25 & 60.11 & 60.47 & 103.86 & 105.25 \\
\hline $61-80$ & 70 & 138 & 41.99 & 44.18 & 57.81 & 57.50 & 96.98 & 98.33 \\
\hline MEAN & 55 & 344 & 43.09 & 44.82 & 59.20 & 59.02 & 101.43 & 102.98 \\
\hline $20-60$ & & & $-1.30 \%$ & $-0.10 \%$ & $0.10 \%$ & $-2.20 \%$ & $1.50 \%$ & $2.20 \%$ \\
\hline $40-80$ & & & $4.60 \%$ & $2.40 \%$ & $3.80 \%$ & $4.90 \%$ & $6.60 \%$ & $6.60 \%$ \\
\hline
\end{tabular}

Table 3. Summary of Estimated Renal Parameters

\begin{tabular}{|l|l|l|l|l|l|l|l|r|}
\hline Age & Age & Sex & $\mathrm{RV}_{\mathrm{R}}$ & $\mathrm{RV}_{\mathrm{L}}$ & $\mathrm{VeC}_{\mathrm{R}}$ & $\mathrm{VeC}_{\mathrm{L}}$ & $\mathrm{RSI}_{\mathrm{R}}$ & \multicolumn{1}{l}{$\mathrm{RSI}_{\mathrm{L}}$} \\
\hline Range & Yrs. & $\mathrm{M} / \mathrm{F}$ & $\mathrm{cm}^{3}$ & $\mathrm{~cm}^{3}$ & & & & \\
\hline Male & Mean & Sample & \pm 26.96 & \pm 25.72 & & & & \\
\hline $21-40$ & 33 & 107 & 155.53 & 159.54 & 0.5287 & 0.5289 & 1.02 & 1.02 \\
\hline $41-60$ & 53 & 122 & 152.59 & 158.00 & 0.5276 & 0.5303 & 1.00 & 1.01 \\
\hline $61-80$ & 70 & 59 & 128.27 & 133.97 & 0.5288 & 0.5301 & 0.99 & 1.01 \\
\hline MEAN & 49 & 288 & 146.74 & 151.76 & 0.5283 & 0.5297 & 1.00 & 1.01 \\
\hline \multirow{2}{*}{ DIV. } & & & $1.89 \%$ & $0.97 \%$ & & & & \\
\cline { 4 - 6 } & & & $15.64 \%$ & $15.20 \%$ & & & & \\
\hline Female & Mean & & \pm 27.08 & \pm 27.78 & \pm 0.0203 & \pm 0.0187 & \pm 0.01 & \pm 0.02 \\
\hline $21-40$ & 34 & 68 & 147.38 & 153.55 & 0.5257 & 0.5316 & 1.02 & 1.03 \\
\hline $41-60$ & 53 & 135 & 145.97 & 153.83 & 0.5277 & 0.5322 & 1.02 & 1.02 \\
\hline $61-80$ & 70 & 122 & 124.54 & 132.26 & 0.5289 & 0.5300 & 0.97 & 0.98 \\
\hline MEAN & 55 & 325 & 142.04 & 148.29 & 0.5280 & 0.5304 & 1.00 & 1.01 \\
\hline \multirow{2}{*}{ DIV. } & & & $0.96 \%$ & $-0.18 \%$ & & & & \\
\cline { 4 - 5 } & & & $14.67 \%$ & $14.02 \%$ & & & & \\
\hline
\end{tabular}

The Left renal size was significantly larger than the right renal size in both genders. Relationship between mean renal lengths was significant when correlated with renal size in term of age and gender. Renal volumes also show much better relationship with renal size based on age and gender. Significant of the relationships between these parameters are shown in the graphical representation in section 5 .

The study shows that male renal size starts early decline before age 60years than female but at a slow rate while female renal size decline after age 60 years but with a faster rate as shown in detail primary raw data Table 2 in appendix. In addition, the overall age and gender renal size reduces significantly after age 80 years as shown by the graphical relationship in figure $\mathbf{1 1}$ and $\mathbf{1 2}$. There is however very little variation between age group 20-40 
and 41-60 years, but significant between age group 4160 and $61-80$ years. Furthermore, both genders in the visual indicators and graphical representation shows similar variation. Generally, the study shows that the male renal parameters is larger than the female renal parameters. The general renal size reductions were extremely significantly with left and females kidneys. The reductions for renal volume were $1.89 \%$ between the age range of $20-40$ to $41-60$ years and $15.64 \%$ between the age ranges of 41-60 to 61-80 years for the right kidney. Similar observations were seen in the left kidney. However, the reductions were pronounce in the female kidneys as shown in the summarized Table 4.2 and Table 4.3. There is very little difference between the left and the right renal size (renal volume, renal length, renal width and renal thickness) as shown in figure 3 to 8 . As a result relative renal length and volume shows approximately $50 \%$ in both right and right with length and right kidney. This is a great renal development factor that are used for clinical decision. The male mean estimated values were 0.4959 and 0.5041 for right renal length and renal volume respectively. While the female average relative left renal length and renal volume were 0.4919 and 0.5081 respectively.

\section{CONCLUSION}

All the measured renal dimensions were within the theoretical range of accepted values, out of the three parameter, the longitudinal diameter was found to be the largest, this was followed by transverse diameter and then the A-P diameter was consider to be the smallest. Indeed, there was a positive correlation between these parameters. The study unveil the fact that renal size (length, width and thickness) diminishes with aging, this is due to parenchymal reduction in the elderly.

The findings of this study suggest that the anatomical description based on 3D CT models could provide evaluation of the anatomic characteristics of the kidneys of potential live kidney donors. The renal volume data in the form of 3D volumetric analysis of CT data as shown could be a promising alternative to nuclear renography in potential kidneys donors in terms of anatomical description. The findings also conclude that the results could be used to study renal development in Ghana.

\section{ACKNOWLEDGEMENTS}

My sincere thanks goes to the entire staff of the Medical Radiation Physics Centre, Radiological and Medical Sciences Research Institute of Ghana Atomic Energy Commission, Department of Radiology, Korle-Bu Teaching Hospital, Tamale Teaching Hospital, Komfo Anokye Teaching Hospital, Cocoa clinic and 37 Military Hospital, for their support and cooperation.

\section{REFERENCES}

[1]. Hendee W R. Physics and applications of medical imaging. Rev Mod Phys. 1999; 71: 444-50.

[2]. Tommy Löfstedt, Olof Ahnlund, Michael Peolsson, and Johan Trygg, Dynamic ultrasound imaging: A multivariate approach for the analysis and comparison of time-dependent musculoskeletal movements, BMC Med Imaging. PMCID: PMC3570433, 10.1186/1471-2342-1229. 2012

[3]. Geraghty EM1, Boone JM, McGahan JP, Jain K, Normal organ volume assessment from abdominal CT. EPUB Abdom Imaging. 2004 Jul-Aug; vol 29 issue 4, pages: 482-90.

[4]. Link F, Kuhagen S, Boskamp T, Rexilius J, Dachwitz S, and Peitgen HO. A Flexible Research and Development Platform for Medical Image Processing and Visualization. Proc. RSNA2004, Chicago, Dec 2004.

[5]. Rexilius J , Spindler W, Jomier J , König M, Hahn HK, Link F, and Peitgen HO. A Framework for Algorithm Evaluation and Clinical Application Prototyping using ITK. Insight Journal as part of the ISC/NA-MIC/MICCAI Workshop on OpenSource Software 2005.

[6]. ICRP, Basic Anatomical and Physiological Data for Use in Radiological Protection Reference Values. ICRP Publication 89. Ann. ICRP 32 2002, pages: $3-4$.

[7]. R. $\mathrm{Xu}$ and Y. W. Chen, "Generalized Ndimensional principal component analysis (GNDPCA) and its application on construction of statistical appearance models for medical volumes with fewer samples," Neurocomputing, vol. 72, no. 10-12, 2009, pp. 2276-2287.

[8]. Bernhard Glodny, Verena Unterholzner, Bernadette Taferner, Karin J Hofmann, Peter 
Rehder, Alexander Strasak and Johannes Petersen. Normal kidney size and its influencing factors - a 64-slice MDCT study of 1.040 asymptomatic patients, BMC Urology, 2009, pages: 9-19

[9]. Seyed Alireza Emamian, Michael Bachmann Nielsen, Jan Fog Pedersen, Lars Ytte. Kidney Dimensions at Sonography: Correlation with Age, Sex, and Habitus in 665 Adult Volunteers. AJR: 160, January 1993, pages: 83-86.

[10]. Jeannette Bakker, Marco Olree, Robert Kaatee, Eduard E. de Lange, Karel G.M. Moons, Jaap J. Beatler and Fredrik J. A. Beek. Renal volume measurement: accuracy and repeatability of US compared with that of MRI imaging. Radiology number 211, vol 3, June 1999 pages: 623-628

[11]. Kiw-Yong Kang, Young Joon Lee, Soon Chul Park, Chul Woo Yang, Yong-Soo Kim, In Sung Moon, Yong Bok Koh, Byung Kee Bang and Bum Soon Choi. A comparative study of methods of estimating kidney length in kidney transplantation donors, Oxford university press, vol 22 (8) pages: 2322-2327 April 23, 2007.

[12]. Sahni D, Jit I, Sodhi L. Weight and measurements of kidneys in northwest Indian adults. Am J Hum Biol. 2001; 13: 726932. International Journal of Collaborative Research on Internal Medicine \& Public Health Vol. 4 No. 9 (2012) 1636.

[13]. Emamian SA, Nielsen MB, Pedersen JF. Intraobserver and interobserver variations in Sonographic measurements of kidney size in adult volunteers. A comparison of linear measurements and volumetric estimates. Acta Radiol. 1995; 36: 3999401.

[14]. Rodney H. Breau, Edward Clark, Bryan Bruner, Patrick Cervini, Thomas Atwell, Greg Knoll, and Bradley C. Leibovich, A simple method to estimate renal volume from computed tomography. Canadian Urol Assoc J. 2013 MayJun; 7(5-6): 189-192.

[15]. Muto NS, Kamishima T, Harris AA, Kato F, Onodera Y, Terae S, Shirato H., Renal cortical volume measured using automatic contouring software for computed tomography and its relationship with BMI, age and renal function, Eur J Radiology. 2011 April, vol 78 issue 1, pages: 151-6.

[16]. Rodney H. Breau, Edward Clark, Bryan Bruner, Patrick Cervini, Thomas Atwell, Greg Knoll, and
Bradley C. Leibovich, A simple method to estimate renal volume from computed tomography. Canadian Urol Assoc J. 2013 MayJun; 7(5-6): 189-192.

[17]. Emamian SA, Nielsen MB, Pedersen JF. Intraobserver and interobserver variations in Sonographic measurements of kidney size in adult volunteers. A comparison of linear measurements and volumetric estimates. Acta Radiol. 1995; 36: 3999401.

[18]. Bernhard Glodny, Verena Unterholzner, Bernadette Taferner, Karin J Hofmann, Peter Rehder, Alexander Strasak and Johannes Petersen. Normal kidney size and its influencing factors - a 64-slice MDCT study of 1.040 asymptomatic patients, BMC Urology, 2009, pages: 9-19

[19]. AAPM Report No.23, "The measurement, Reporting, and Management of Radiation Dose in CT”, 2008, Diagnostic Imaging Committee, ISBN: 978-1-888340-73-0 ISSN: 0271-7344. Page 11.

[20]. McAfee JG., Cloutier RJ, Edwards CL, Snyder WS, eds. Problems in evaluating the radiation dose for radionuclides excreted by the kidneys. Medical Radionuclides: Radiation Dose and Effects. Oak Ridge, U.S. Atomic Energy Commission; 1969, pages: 271-294.

[21]. Janoff DM1, Davol P, Hazzard J, Lemmers MJ, Paduch DA, Barry JM., Computerized tomography with 3-dimensional reconstruction for the evaluation of renal size and arterial anatomy in the living kidney donor. J Urol. 2004 Jan; 171(1) pages: $27-30$.

[22]. Janoff DM1, Davol P, Hazzard J, Lemmers MJ, Paduch DA, Barry JM., Computerized tomography with 3-dimensional reconstruction for the evaluation of renal size and arterial anatomy in the living kidney donor. J Urol. 2004 Jan; 171(1) pages: $27-30$.

[23]. Lerner LB, Henriquez HF, and Harris RD, Interactive 3-dimensional computerized tomography reconstruction in evaluation of the living renal donor, journal of urology, March 1999, vol 161 issue 2, pages: 403-407.

[24]. Ho Sik Shin, Byung Ha Chung, Sang Eun Lee, Woo Jin Kim, Hong Il Ha and Chul Woo Yang. Measurement of Kidney Volume with MultiDetector Computed Tomography Scanning in 
Young Korean Yonsei Med J 50(2), pages: 262265, 2009.

[25]. Bernhard Glodny, Verena Unterholzner, Bernadette Taferner, Karin J Hofmann, Peter Rehder, Alexander Strasak and Johannes Petersen. Normal kidney size and its influencing factors - a 64-slice MDCT study of 1.040 asymptomatic patients, BMC Urology, 2009, pages: 9-19

[26]. Geraghty EM1, Boone JM, McGahan JP, Jain K, Normal organ volume assessment from abdominal CT. EPUB Abdom Imaging. 2004 Jul-Aug; vol 29 issue 4, pages: 482-90.

[27]. Th. Scholbach and D.Weitzel, Body-Surface-Area Related Renal Volume: A Common Normal Range from Birth to Adulthood, Hindawi Publishing Corporation Scientifica, Volume 2012, Article ID 949164

[28]. V. T. Ninan, K. Thomas Koshi, M. M. Niyamthullah, C. K. Jacob, G. Gopalakrishnan, A. P. Pandey and J. C. M. Shastry. A Comparative Study of Methods of Estimating Renal Size in Normal Adults, Oxford Journals Medicine \& Health, Nephrology Dialysis Transplantation, Volume 5, Issue 10, Pp. 851-854.

[29]. Orhan Ozbek, Yalcin Solak, Ibrahim Guler, Huseyin Ozbiner, Seda Ozbek, Kultigin Turkmen, Alaaddin Nayman, Zeynep Biyik, Cigdem Samur, Suleyman Turk, Predictors of kidney dimensions measured by multi-detector computed tomography (MDCT) in 930 middle-aged and elderly patients. Ren Fail 2012. Epub 2011 Nov 29. 29:34 (1):53-9 